\title{
La calidad de la enseñanza en la Educación Superior en Ecuador
}

\section{The quality of teaching in higher education in Ecuador}

\section{A qualidade do ensino no ensino superior no Equador}

\author{
Gabriela Rossana Guerreo-Aray ${ }^{\mathrm{I}}$ \\ gguerrero@utm.edu.ec \\ Silvia Monserrate Suástegui-Solórzano "I \\ ssuastegui@utm.edu.ec \\ Gabriela Esperanza Zambrano-Vera III \\ gezambrano@utm.edu.ec
}

Recibido: 23 de marzo de 2019 *Aceptado: 17 de junio de 2019 * Publicado: 05 de julio de 2019

I. Magíster en Gerencia Educativa, Licenciada en Ciencias de la Educación General Básica, Facultad de Filosofía Letras y Ciencias de la Educación, Departamento de Filosofía, Universidad Técnica de Manabí, Portoviejo, Ecuador.

II. Magíster en Gerencia Educativa, Diploma Superior en Liderazgo Educativo, Ingeniero Comercial,

Facultad de Filosofía Letras y Ciencias de la Educación, Departamento de Didáctica, Universidad Técnica de Manabí Portoviejo, Ecuador.

III. Magíster en Pedagogía, Licenciada en Ciencias de la Educación Mención Inglés, Facultad de Filosofía Letras y Ciencias de la Educación, Área de Pedagogía, Universidad Técnica de Manabí Portoviejo, Ecuador. 


\section{Resumen}

Particularmente para América Latina, la educación de calidad es uno de los factores que mayores desafíos ha representado en la región. El inevitable proceso de integración a un mundo cada vez más interconectado y globalizado, que va de la mano con cada una de las particulares demandas sociales, exponen a cada país de la región a una relativa presión que, aunado al punto de inflexión por el que atraviesa el desarrollo económico de cada nación, hace que el cambio educativo sea visto como una estrategia prioritaria. Distintivamente desde 2008, Ecuador no ha sido la excepción en cuanto al avance en la calidad de la enseñanza de su Sistema de Educación Superior, no obstante, se vuelve interesante conocer, en términos prácticos, en qué situación se encuentra este indicador. Este contexto es lo que motivó el desarrollo del presente trabajo, particularmente enfocado a la búsqueda y revisión de literatura vigente que de una manera clara sirva para exponer: ¿cómo es considerada, recientemente, la calidad de la enseñanza en la educación superior ecuatoriana? En conclusión, se llega a defender la idea de que la calidad de enseñanza a nivel superior, primordialmente dependerá del docente, quien durante el transcurso en el que este impartiendo y facilitando conocimientos, debe ser constantemente evaluado, a fines de velar que el mismo vaya desarrollo las competencias y destrezas idóneas como insumos complementarios del estándar profesional, y así, procurar garantizar la necesaria adaptación de éstos a las exigencias de un entorno globalizado, y más aún, del propio medio social, económico, cultural, político y geográfico del que es responsablemente protagonista; aspecto éste en el que particularmente el sistema de educación superior ecuatoriano, mantiene un lento (aún) pero constante logro de metas.

Palabras clave: estrategia; indicador; docente; competencias; profesional.

\section{Abstract}

Particularly for Latin America, quality education is one of the biggest challenges in the region. The inevitable process of integration into an increasingly interconnected and globalized world, which goes hand in hand with each of the particular social demands, exposes each country in the region to a relative pressure that, together with the turning point by which it crosses the economic development of each nation, it makes educational change seen as a priority strategy. Distinctively since 2008, Ecuador has not been the exception in terms of progress in the quality of education of

\footnotetext{
259 Vol. 5, núm. 3, julio, 2019, pp.258-270

Gabriela Rossana Guerreo Aray, Silvia Monserrate Suástegui Solórzano, Gabriela Esperanza Zambrano Vera
} 
its Higher Education System, however, it becomes interesting to know, in practical terms, in what situation this indicator is. This context is what motivated the development of this work, particularly focused on the search and review of current literature that clearly serves to expose: how is the quality of teaching in Ecuadorian higher education considered recently? In conclusion, we come to defend the idea that the quality of higher education, primarily depend on the teacher, who during the course in which this imparting and facilitating knowledge, must be constantly evaluated, in order to ensure that it goes develop the competencies and skills as complementary inputs to the professional standard, and thus seek to ensure the necessary adaptation of these to the demands of a globalized environment, and even more, the social, economic, cultural, political and geographical environment of which it is responsibly protagonist; this aspect in which particularly the Ecuadorian higher education system, maintains a slow (yet) but constant achievement of goals.

Key words: strategy; indicator; teacher; skills; professional.

\section{Resumo}

O Particularmente para a América Latina, a educação de qualidade é um dos maiores desafios da região. O inevitável processo de integração em um mundo cada vez mais interconectado e globalizado, que anda de mãos dadas com cada uma das demandas sociais particulares, expõe cada país da região a uma pressão relativa que, juntamente com o ponto de atravessa o desenvolvimento econômico de cada nação, faz da mudança educacional vista como uma estratégia prioritária. Distintamente desde 2008, o Equador não tem sido a exceção em termos de progresso na qualidade da educação de seu Sistema de Ensino Superior, no entanto, torna-se interessante saber, em termos práticos, em que situação está esse indicador. Este contexto é o que motivou o desenvolvimento deste trabalho, particularmente focado na busca e revisão da literatura atual que claramente serve para expor: como é a qualidade do ensino no ensino superior equatoriano considerado recentemente? Em conclusão, chegamos a defender a ideia de que a qualidade do ensino superior, depende principalmente do professor, que durante o curso em que esta transmitir e facilitar o conhecimento, deve ser constantemente avaliado, a fim de garantir que ele vai desenvolver as competências e habilidades como insumos complementares ao padrão profissional, e assim buscar assegurar a necessária adaptação destes às demandas de um ambiente globalizado, e ainda mais, ao

\footnotetext{
260 Vol. 5, núm. 3, julio, 2019, pp.258-270

Gabriela Rossana Guerreo Aray, Silvia Monserrate Suástegui Solórzano, Gabriela Esperanza Zambrano Vera
} 
meio social, econômico, cultural, político e geográfico de que é originário. responsavelmente protagonista; este aspecto em que particularmente o sistema de ensino superior equatoriano, mantém um lento (ainda), mas constante, cumprimento de metas.

Palavras-chave: estratégia; indicador; professor; habilidades; profissional.

\section{Introducción}

Es pertinente iniciar refiriendo el trabajo investigativo de Naranjo (2017) quien citando a (Segrera, 2016) rescata la trascendente premisa que surgió desde hace más de 20 años, resultado de las sugerencias de la UNESCO y las Comisiones de Seguimiento en la Conferencia Mundial sobre la Educación Superior de 1998. Las mismas estaban referidas a la necesaria y constante actualización por parte del docente, pero también de los contenidos, los currículos, y de las redes electrónicas en pro del aprendizaje; sugerencias que igualmente se evidenciaban de las contribuciones científicas más importantes. El autor continúa afirmando que tal proposición tuvo una especial acogida en países como Chile, Argentina y Ecuador, y cita a (Lamarra, 2012) para referir que es quien deja evidencia de ello cuando aseguró que fue:

[...] necesario establecer procesos de regulación de la educación superior. A través de procesos tendientes a la acreditación institucional [...], se ha posibilitado limitar la proliferación excesiva de nuevas instituciones universitarias y tener a una mayor homogeneidad en cuanto a los niveles de calidad. (Naranjo, 2017, p. 2)

Cobos (2018), mediante su obra ha afirmado que, los principios fundamentales en los que se enmarca el enfoque el sistema de educación superior ecuatoriano contemporáneo, están: los del “cultivo del espíritu, la moral, los valores" y, recientemente, el desarrollo de competencias y destrezas que conllevan a la ampliación juicios reflexivos y críticos en pro de "soluciones a los problemas de la sociedad", que en conjunto favorecen la ocasión para el logro de anhelos y objetivos tanto individuales como colectivos. Asegura que:

La educación universitaria permite de forma amplia el cambio en el progreso de los países y en el acrecentamiento de la calidad de vida de sus pueblos. Es uno de los medios culturales que admite a las naciones a convivir en un constante proceso de interacción con su medio descubriendo y 
cultivando su individualidad y fortaleciendo su convivencia en colectividad [...] (Cobos, 2018, p. 29-30)

Claro, es posible que lo antes expuesto, bien puede estar fundamentado en la Ley Orgánica de Educación Superior (LOES) vigente desde el 2010, ya que en su artículo 12 claramente se establece que:

El Sistema de Educación Superior se rige por los principios de autonomía responsable, cogobierno, igualdad de oportunidades, calidad, pertinencia, integridad y autodeterminación para la producción del pensamiento y conocimiento en el marco del diálogo de saberes, pensamiento universal y producción científica tecnológica global. (LOES, 2010).

En base al marco contextual antes descrito, se establece como objetivo central explorar sobre la concepción que otros especialistas tienen sobre la calidad de la enseñanza en la educación superior ecuatoriana recientemente, y para ello se hará referencia descriptiva del análisis interpretativo deducido del conjunto de material bibliográfico seleccionado, a fines de comprender dicho tema, y a su vez, de que el presente material propicie la iniciativa de nuevos debates e investigaciones.

\section{Materiales y Métodos}

Las herramientas y materiales de trabajo, primordialmente, son computadores personales, libros, trabajos de grado, tesis de postgrado y doctorado, folletos, revistas científicas, boletines, informes, prensa escrita, entre otros, con los que se ejecuta parte de la metodología inherente a una investigación científica de tipo documental.

La presente investigación limita su enfoque a la búsqueda y revisión sistemática de material bibliográfico accesible tanto de manera física como a través del uso de diversas bases de datos, entre las que destacaron la de: SciELO, Dialnet, Redalyc, Alicia, Base, Redib, Refseek, entre otras; en las que igualmente se fue escogiendo material bibliográfico de la misma categoría antes descrita, y además, artículos científicos, ensayos, revisiones sistemáticas, protocolos, editoriales, noticias científicas y otros tipos de documentos e información de interés científico y académico, destacando todo eso como parte de la materialización del proceso de investigación documental, en la que de

262 Vol. 5, núm. 3, julio, 2019, pp.258-270

Gabriela Rossana Guerreo Aray, Silvia Monserrate Suástegui Solórzano, Gabriela Esperanza Zambrano Vera 
hecho se leyó, analizó, discutió el cúmulo de la literatura seleccionada para así alcanzar la comprensión que facilita la síntesis de la mejor evidencia disponible, siendo esto por cierto uno de los resultados esperados.

La búsqueda en las distintas bases de datos es aleatoria y consecutiva, usando las expresiones "calidad de la enseñanza en la educación superior ecuatoriana", "calidad de la enseñanza en la educación superior+Ecuador" y "Calidad de la enseñanza superior+Ecuador"; siendo del gran número de resultados arrojados por éstas que se aplican los filtros de: idioma (Español-Castellano) y año de publicación (2013 -2019, con algunas excepciones por la relevancia del tema), básicamente; y en definitiva seleccionando más de treinta documentos que guardaron la mayor semejanza a éste tema.

Al final de éste proceso se procede a rescatar todo el material bibliográfico, tanto en formato digital como físico, y clasificándolo, se efectúa la imprescindible lectura por parte cada miembro del equipo, quienes luego de su particular interpretación, análisis crítico, discusión y comprensión, consensuadamente logran la inclusión de las ideas al desarrollo del presente material, de conformidad a la correlación de éstos para con el objetivo previamente planteado para el presente estudio, y descartando aquellas que no se correlacionaban con el mismo y que, por ende, no sirven de fundamento los planteamientos aquí expresados.

\section{Resultados}

\section{Calidad educativa}

La calidad educativa desde un punto de vista organizacional es el " $[\ldots]$ conjunto de factores que hacen referencia a todos los ámbitos de la organización. Pero también, la calidad debe ser entendida como algo que es susceptible de mejorar día a día" (ISOTools, 2017)

Por su parte, Véliz (2018) se fundamenta en las ideas de (González \& Espinoza, 2008) y (Días, 2012) para referir en su obra que coincide con estos últimos en el hecho de que la razón del incremento en cuanto a la calidad de la educación superior, ya no puede ser concebido como "un proceso unilineal que puede ser evaluado por un conjunto de indicadores estáticos y cuantitativos",

263 Vol. 5, núm. 3, julio, 2019, pp.258-270

Gabriela Rossana Guerreo Aray, Silvia Monserrate Suástegui Solórzano, Gabriela Esperanza Zambrano Vera 
sino todo lo contrario, debe ser vista como "un proceso multifactorial" cuyos efectos se diferencian en proporción de particulares patrones de referencia.(p. 2)

Tratadistas como Flores \& Pernía (2018) han asegurado que a pesar de que en el Ecuador se han alcanzado grandes logros en materia de calidad, inclusión y pertinencia de la educación superior, ese sistema "aún conserva profundas brechas que reproducen las características estructurales de la asimetría económica y poblacional del país” (p. 218). Posteriormente afirman que “[...] la educación de calidad se constituye como uno de los elementos centrales de la democratización de derechos, como primer paso para construir una sociedad más productiva” (Flores \& Pernía, 2018, p. 225)

\section{Calidad de la enseñanza}

Hace relativamente poco, una iniciativa renovadora se ha elevado mediante un informe producido por la Comisión para la Educación de Calidad para Todos, perteneciente a la organización The Inter-American Dialogue, el cual promueve "un cambio educativo profundo en América Latina y el Caribe a través de la movilización de los sectores público y privado, los medios de comunicación y la sociedad civil". En el informe, basado en el minucioso análisis del estado de los sistemas educativos de la mencionada región, se demuestra que "los niños y jóvenes latinoamericanos no están aprendiendo a niveles aceptables y que América Latina se sigue distanciando con respecto al resto del mundo desarrollado y emergente", es por ello que dicha Comisión "recomienda acciones en seis áreas prioritarias para mejorar la calidad educativa” entre las que destacan:

$1^{\mathrm{o}}$. Asentar mejor las bases del aprendizaje mediante la inversión en educación temprana, por cuanto existe suficiente evidencia científica que demuestra que las habilidades fundamentales que definen la capacidad de aprender, aunque parezca paradójico, se desarrollan temprano en nuestras vidas, es decir, previas al comienzo de la escolaridad primaria.

$2^{\circ}$. Promover la excelencia docente mediante el constante mejoramiento de su desempeño, considerándose a la hora de formular las políticas y prácticas conducentes a tales fines, trascendentales aspectos tales como: la formación inicial (incluyendo quienes deciden estudiar para

\footnotetext{
264 Vol. 5, núm. 3, julio, 2019, pp.258-270

Gabriela Rossana Guerreo Aray, Silvia Monserrate Suástegui Solórzano, Gabriela Esperanza Zambrano Vera
} 
ser docentes), la carrera (incluyendo los mecanismos de entrada, promoción, reconocimiento y remuneración), los sistemas de apoyo a docentes (incluyendo la formación profesional), y la gestión (incluyendo evaluación para el mejoramiento continuo).

$3^{\circ}$. Evaluar el aprendizaje, fundamental para establecer estándares altos que fijen expectativas claras, tanto para padres y alumnos como para docentes y administradores, y también, para guiar las acciones en pro de la mejoría la calidad educativa, de allí la importancia de profundizar en los esfuerzos por comunicar los resultados y usarlos para la gestión en todos los niveles.

4. Implementación de nuevas tecnologías, que se trata más que la interpretación simplista de incorporar computadoras, sino más bien como instrumentos de apoyo pedagógico en el marco de una modernización en la formación docente y la gestión del aula que en definitiva influyan en el aprendizaje.

$5^{\circ}$. Fomento de la educación relevante, pues en la región se arrastra con la falta de relevancia de los programas de estudio, que afecta la habilidad de los graduados para desenvolverse en un mundo complejo y crecientemente globalizado, lo que en concreto debe entenderse la modernización de la educación media y terciaria para promover el desarrollo de competencias demandadas por los empleadores, que a su vez implica ampliar la mirada acerca de qué constituye una educación de calidad, resaltando la relevancia de los aprendizajes adquiridos y las competencias desarrolladas.

$6^{\circ}$. Procurar el financiamiento sustentable, en términos fiscales y socialmente. América Latina todavía hay varios países en los que el sector educativo no recibe suficiente prioridad a la hora del reparto de recursos. En ellos se necesita un mayor esfuerzo y compromiso público con el financiamiento a la educación. Al mismo tiempo, la eficiencia en el uso y la asignación de los recursos es un desafío generalizado en los sistemas educativos de la región. (Comisión para la Educación de Calidad para Todos, 2016)

\footnotetext{
265 Vol. 5, núm. 3, julio, 2019, pp.258-270

Gabriela Rossana Guerreo Aray, Silvia Monserrate Suástegui Solórzano, Gabriela Esperanza Zambrano Vera
} 
Como corolario a lo antes expuesto, bien cabe mencionar el trabajo de Jerez, Orsini, \& Hasbún (2016), quienes con su aporte rescatan, mediante la referencia hecha a los aportes de (Bain, 2004; Hannah et al., 2011) que con anterioridad se tenía evidencia que demostraba "que los estudios sobre calidad de la docencia universitaria cobran relevancia académica para aumentar la comprensión, proyección y mejoramiento continuo del sistema universitario, y que este tipo de estudios requieren de los contextos particulares de cada sistema educativo.”(p. 502)

\section{Enseñanza en la educación superior}

De acuerdo con Hernández \& Infante (2017), quien a su vez apoyándose en la idea de (Didriksson, 2003), indica que a éste nivel, el proceso de enseñanza-aprendizaje debe estar concebido por el docente en relación a la formación de los estudiantes como futuros profesionales, ya que estos deben irse instruyendo con la finalidad de adquirir las capacidades para identificar los problemas asociados a su carrera, y, por ende, poder proponer las respectivas soluciones, siendo con esa manera de proceder que entonces, el docente le es posible dirigir la enseñanza-aprendizaje a nivel universitario con un enfoque profesional, en función del desarrollo de modos de actuación en el estudiante.

\section{Realidad ecuatoriana}

El caso ecuatoriano en cuanto a la educación superior ha sido expuesto de manera sucinta por Tornay (2016), quien asevera que éste sistema viene de ser rescatado de manos privadas que además no contaba con un ente regulador, escenario que facilitó su degradación, característicamente por aquellas instituciones que, inclusive "bajo modalidades de negocio familiar", respaldaban titulaciones académicas de mediocre valor.

Asegura que un hecho que marcó la ruptura de esta situación fue la aprobación de la Constitución en 2008 y la puesta en marcha del Plan Nacional del Buen Vivir, iniciativa que protagonizó una apremiente modernización de varios sectores estratégicos, siendo uno de los principales, precisamente el educativo, en aras de superar el atraso endémico que azotaba al país. Culmina ilustrando el panorama describiendo que, luego de un penoso lustro de reportar "los peores índices

\footnotetext{
266 Vol. 5, núm. 3, julio, 2019, pp.258-270

Gabriela Rossana Guerreo Aray, Silvia Monserrate Suástegui Solórzano, Gabriela Esperanza Zambrano Vera
} 
de América Latina en distintas categorías" fue con la aprobación de la Ley Orgánica de Educación Superior (LOES), a escasos tres años de iniciar el nuevo plan de gobierno, que Ecuador se enrumba para situarse a la vanguardia de la región en materia educativa, siendo en base al referido ordenamiento jurídico que se crean organismos tales como el Consejo de Educación Superior (CES) y el Consejo de Evaluación, Acreditación y Aseguramiento de la Calidad de la Educación Superior (CEAACES), que en lo sucesivo comenzarían a regir el novedoso Sistema de Educación Superior. La autora, a manera de ejemplo, hace especial énfasis en la función de uno de los mencionados organismos, exponiendo que:

Entre las tareas más urgentes que asume el CEAACES, y que a día de hoy continúa creando controversia, se sitúa la acreditación de las universidades en distintas categorías en función de los avances obtenidos en diversas áreas, como infraestructuras, investigación, publicaciones académicas, cualificación docente o vinculación con la comunidad, entre otras. El modelo de acreditación suspendió a las conocidas como "universidades de garaje" y, en el momento actual, obliga a las instituciones de educación superior a la superación de evaluaciones periódicas. En caso de no aprobar, las entidades pierden la concesión de su licencia, o bien, son obligadas a eliminar de su oferta a las carreras que no superen los parámetros. (Tornay, 2016)

Ya con la descripción de ésta especialista se puede evidenciar que las autoridades ecuatorianas emergentes, no solo estaban al tanto de la complejidad de la problemática que padecía el Sistema Educativo Superior, sino que incluso tenían definidas las acciones pertinentes que debían ejecutar para el rescate de dicho sistema, y claramente se deja ver que la calidad de enseñanza, es uno de sus objetivos primordiales.

Otros puntos de vista de dicha realidad ofrecen Barrera, Barragán, \& Ortega (2017), ya que desarrollan el tema desde la perspectiva del docente. Dicen que, independientemente de que a partir del mes de noviembre de 2012 en el Ecuador se haya aprobado mediante un Acuerdo Ministerial la aplicación de los mismos estándares de calidad educativa que son aplicados, por ejemplo, en Hong Kong desde 1980; y por cierto ha derivado en un sistema de constante mejora a raíz de una permanente evaluación ejecutada desde sus inicios; no hay evidencia de que los mismos hayan

\footnotetext{
267 Vol. 5, núm. 3, julio, 2019, pp.258-270

Gabriela Rossana Guerreo Aray, Silvia Monserrate Suástegui Solórzano, Gabriela Esperanza Zambrano Vera
} 
tenido "una verdadera aplicación ni evaluación”, pues aunque hayan sido proyectados con la mejor de las intenciones, dicho proceso no ha sido "el correcto porque no hay un verdadero diagnóstico de la calidad de nuestra educación", no existen datos destacados que de alguna manera denoten cual es la verdad de los aprendizajes, por dura que sea, y eso aunado al hecho de que tampoco se evidencia el compromiso de un considerable número docentes, porque esa función en recientemente, se ha convertido en la de un "profesional de escritorio, que tiene que pasar horas y horas, escribiendo, planificando, diseñando evaluaciones, preparando sus clases y adaptando la metodología que se aplica en cada una de las aulas", contexto que notoriamente es contrario con la calidad de enseñanza que necesita la educación ecuatoriana. (p. 11)

\section{Seguidamente los tratadistas destacan que:}

La calidad es un proceso continuo de trabajo colaborativo y organizado en donde todos los miembros forjan efectos positivamente valorados por la sociedad respecto del proceso de formación y estos son enfocados al aprendizaje, al desempeño profesional, a la infraestructura y a la gestión educativa, en donde la dimensión y los dominios de cada estándar deben ser cumplido con efectividad. (p. 11)

De lo anterior, es fácil comprender el punto de vista que los autores tratan de dar a entender, y no es otro que el del trabajo conjunto entre los actores en el proceso de formación (básicamente docente y estudiante) para poder lograr las competencias necesarias con la calidad requerida; no obstante, más adelante aseveran que, al respecto, la realidad en el Ecuador desde la perspectiva del docente como actor directo de la formación estudiantil ha sido distinta pues, en los últimos años, ha sido incierta la idea de enseñar y aprender ya que ha arrastrado una cadena cambios que, en sus palabras, "[...] no han beneficiado a la enseñanza ecuatoriana. La revolución educativa de la cual tanto se habla, no se ha podido evidenciar en los salones de clase" y ello ha sido porque "[...] en realidad no existe una coherencia en los planes de estudio, entre la Educación Inicial, la Educación Básica, el Bachillerato y la Universidad” (p. 11)

\footnotetext{
268 Vol. 5, núm. 3, julio, 2019, pp.258-270

Gabriela Rossana Guerreo Aray, Silvia Monserrate Suástegui Solórzano, Gabriela Esperanza Zambrano Vera
} 
En definitiva, los resultados de la investigación efectuada por éstos especialistas dejan en evidencia que son muchos los significados que definen la realidad educativa ecuatoriana desde la perspectiva del docente, razones por las que no existe una variable inequívoca de tal situación sino un cúmulo de conceptualizaciones pedagógicas complejas sobre lo que es la práctica educativa "desde la estructura del sistema ecuatoriano de calidad, los perfiles de ingreso y egreso de los diferentes niveles de educación, la tecnología y la investigación; desarrollo de las competencias y los estándares de calidad" (p. 15)

\section{Conclusiones}

Luego de ser estudiada y discutida toda la literatura científica escogida para el desarrollo de este trabajo, es posible atreverse a asegurar que, en síntesis, la calidad de enseñanza a nivel superior, primordialmente, dependerá del docente quien, por una parte; siendo constante y debidamente evaluado, acorde a las exigencias propias de un ineludible entorno globalizado, y más aún, a las del propio medio social, económico, cultural, político y geográfico al que pertenece; es el que en (primera instancia) se encuentra comprometido para consigo mismo y para con sus dependientes, en desarrollar competencias y destrezas idóneas, como insumos complementarios del estándar profesional en la enseñanza, lógicamente en aras de la referida calidad; insumos que a su vez se encuentran asociados a la práctica pedagógica/andragógica, profesionalismo contributivo, medidas de desarrollo colaborativo y el aprendizaje-enseñanza del estudiante, y por la otra, porque es él mismo, el docente, quien hasta última instancia, gozará de la potestad y tendrá la responsabilidad de exigir, explotar y diferencia el potencial, evaluar el rendimiento, orientar y acompañar y colaborar con el estudiante en la búsqueda constante de la excelencia, es decir, de la calidad de su enseñanza.

\section{Referencias Bibliográficas}

Asamblea Nacional. (2010). Ley Orgánica De Educación Superior (Asamblea Nacional ed.). Quito, Pichincha, Ecuador: Registro Oficial. Recuperado el 05 de 07 de 2019

Barrera, H., Barragán, T., \& Ortega, G. (31 de 12 de 2017). La realidad educativa

\footnotetext{
269 Vol. 5, núm. 3, julio, 2019, pp.258-270

Gabriela Rossana Guerreo Aray, Silvia Monserrate Suástegui Solórzano, Gabriela Esperanza Zambrano Vera
} 
ecuatoriana desde una perspectiva docente. 9Revista Iberoamericana de Educación, 75(2), 9-20. Recuperado el 05 de 07 de 2019, de https://rieoei.org/RIE/article/view/2629/3612

Cobos, J. (mayo -dicimbre de 2018). La Educación Superior en el Ecuador. Situación actual y perspectivas de futuro desde el contexto de las Tecnologías de la Información y la Comunicación. International Studies on Law and Education, 29-40. Recuperado el 05 de 07 de 2019, de http://www.hottopos.com/isle29_30/29-40Cobos.pdf

Naranjo, A. D. (2017). congresoalacip2017.org. Recuperado el 05 de 07 de 2019, de congresoalacip2017.org:

http://www.congresoalacip2017.org/arquivo/downloadpublic2?q=YToyOntzOjY6InBhcmFtcyI7 czozNToiYToxOntzOjEwOiJJRF9BUlFVSVZPIjtzOjQ6IjE5OTEiO30iO3M6MToiaCI7czozMj oiZGYwZDMwOTMyYzUzMjk4MmM3ZTA3YWM1NWJjMTQ0YzciO30\%3D

Tornay, M. (17 de 02 de 2016). El nuevo modelo educativo, un camino hacia el buen vivir ecuatoriano. Pueblos, 1. Recuperado el 05 de 07 de 2019, de http://www.revistapueblos.org/blog/2016/02/17/el-nuevo-modelo-educativo-un-camino-hacia-elbuen-vivir-ecuatoriano/

270 Vol. 5, núm. 3, julio, 2019, pp.258-270

Gabriela Rossana Guerreo Aray, Silvia Monserrate Suástegui Solórzano, Gabriela Esperanza Zambrano Vera 